BMJ Open

Sport \&

Exercise

Medicine

\title{
Association of the British Athletic Muscle Injury Classification and anatomic location with return to full training and reinjury following hamstring injury in elite football
}

Ricky Shamji (D) , ${ }^{1,2}$ Steven L J James, ${ }^{3,4}$ Rajesh Botchu, ${ }^{3,4}$ Kent A Khurniawan, ${ }^{4}$ Gurjit Bhogal, ${ }^{1,2}$ Alison Rushton ${ }^{4,5}$

To cite: Shamji R, James SLJ, Botchu R, et al. Association of the British Athletic Muscle Injury Classification and anatomic location with return to full training and reinjury following hamstring injury in elite football. BMJ Open Sport \& Exercise Medicine 2021;7:e001010. doi:10.1136/ bmjsem-2020-001010

Accepted 16 April 2021
Check for updates

(c) Author(s) (or their employer(s)) 2021. Re-use permitted under CC BY-NC. No commercial re-use. See rights and permissions. Published by BMJ.

${ }^{1}$ Medical Department, Aston Villa FC, Birmingham, UK ${ }^{2}$ Centre for Musculoskeletal Medicine, Royal Orthopaedic Hospital, Birmingham, UK ${ }^{3}$ Imaging Department, Royal Orthopaedic Hospital, Birmingham, UK

${ }^{4}$ School of Sport, Exercise and Rehabilitation Sciences, University of Birmingham, Birmingham, UK

${ }^{5}$ School of Physical Therapy, University of Western Ontario, London, Ontario, Canada

Correspondence to Dr Ricky Shamji; ricky.shamji@avfc.co.uk

\section{ABSTRACT}

Background The relationship between hamstring muscle injuries (HMls) that involve the intramuscular tendon and prolonged recovery time and increased reinjury rate remains unclear in elite footballers.

Objective To determine the association of time to return to full training (TRFT) and reinjury of HMls using the British Athletic Muscle Injury Classification (BAMIC) and specific anatomical injury location in elite-level football players. Methods The electronic medical records of all players at an English Premier League club were reviewed over eight consecutive seasons. All players who sustained an acute HMl were included. Two experienced musculoskeletal radiologists independently graded each muscle using the BAMIC, categorised each injury location area (proximal vs middle vs distal third and proximal vs distal tendon) and reported second muscle involvement. TRFT and reinjury were recorded.

Results Out of $61 \mathrm{HMls}$, the intramuscular tendon (BAMIC 'c') was involved in 13 (21.3\%). HMI involving the intramuscular tendon ('C') had a mean rank TRFT of 36 days compared with 24 days without involvement $(p=0.013)$. There were $10(16.4 \%)$ reinjuries with a significant difference of $38.5 \%$ reinjury rate in the group with intramuscular tendon injury (' $c$ ') and $12.5 \%$ in the group without $(\mathrm{p}=0.031)$. TRFT and reinjury involving a second muscle was statistically significantly higher than without. Most of the HMls to the biceps femoris with reinjury (5 out of 9 ) were in the distal third section related to the distal tendon site involving both the long and short head.

Conclusion TRFT in $\mathrm{HMl}$ involving the intramuscular tendon ('c') of the Biceps femoris is significantly longer with significantly higher reinjury rate compared with injuries without, in elite football players. The finding that most reinjures of the biceps femoris occurring in the distal third muscle at the distal tendon site, involving both the long and short head, merits further investigation.

\section{INTRODUCTION}

Hamstring muscle injuries (HMIs) in elite football are common and may result in a

\section{SUMMARY}

What are the new findings?

- Hamstring muscle injuries (HMls) extending into the intramuscular tendon, categorised as British Athletic muscle injury 'c' on MRI, are associated with longer time to return to full training and increased reinjury rate of elite-level players in English football.

- Most of the HMls to the biceps femoris with reinjury were seen at the distal musculotendinous $\mathrm{T}$ junction (DMTJ) involving both the long and short heads on MRI.

How might it impact on clinical practice in the future?

- HMI patterns, beyond intramuscular tendon involvement, such as location and second muscle injury warrants consideration for clinicians managing elitelevel football players.

- The recognition of DMTJ of the biceps femoris injuries may be important in elite-level football.

prolonged absence with a high risk of reinjury. ${ }^{12}$ Hamstring reinjury rates are reported to be between $14 \%$ and $63 \% .^{2}$ Furthermore, Ekstrand et $a l^{3}$ recently reported that HMIs have increased by $4 \%$ annually in mens professional football since 2001. Medical teams face pressure to make an accurate diagnosis and identify important prognostic factors for return to play in the shortest time possible while still ensuring that the risk of injury reoccurrence is minimal.

HMIs involving the intramuscular tendon have been associated with prolonged recovery time and a higher reinjury rate in professional athletes. ${ }^{4-8}$ Pollock et at ${ }^{69}$ used their validated British Athletics Muscle Injury Classification (BAMIC) which distinguishes anatomical site ('a': myofascial; 'b': musculotendinous junction or 'c': intramuscular tendon) and injury severity (grading 0-4) based on MRI 
measurements within muscle or tendon, to carry out a retrospective study of HMIs in track and field athletes. They found BAMIC grade 'c' injuries took significantly time to return to full training (TRFT) and had a reinjury rate of up to $63 \%$ compared with myofascial and musculotendinous injury. Given that clinical examination alone is unable to discriminate the presence of intramuscular tendon involvement, MRI is needed in professional sport. $^{10}$

The first prospective study of 70 HMIs by van der Made $e t a l^{11}$ has put this increased TRFT and reinjury association with intramuscular tendon involvement into doubt. Their study of athletes from various sports does not support the notion that injuries involving the tendon are significantly more serious. They demonstrated that return to play was only slightly over a week longer for HMIs involving the intramuscular tendon vs those that are not $(30.2 \pm 10.8$ vs $22.2 \pm 7.4$ days $)$ and conclude that its clinical relevance for the individual athlete is limited.

Other hamstring injury patterns may also be important. Entwisle $e t a l^{12}$ describe the distal musculotendinous $\mathrm{T}$ junction (DMTJ) of the biceps femoris where the opposing anterolateral aspect of the long head and the posterolateral aspect of the short head form the musculotendinous junction which appears as a T-shaped structure. They report higher reinjury rates involving this DMTJ of biceps femoris muscle in their cross-sectional study of 106 injuries in Australian Football players. The reinjury rate was $54 \%$, with $86 \%$ of subsequent injuries being the same or of higher grade than the index injury.

Differences in study design, sporting demands and failure to recognise distinct clinical entities such as the DMTJ, may contribute to the contrasting reports of HMI studies. The purpose of this study was to determine whether HMIs graded according to the BAMIC were associated with delayed time to return to full training (TRFT) and higher recurrence rates in elite male football players. The study also aimed to assess other hamstring anatomical locations (proximal vs middle vs distal and proximal vs distal tendon) that may be associated with delayed TRFT and higher recurrence rates in elite male football players. Our working hypothesis was that HMIs involving the intramuscular tendon and injuries involving the distal biceps femoris are associated with longer TRFT and higher reinjury rate.

\section{METHODS \\ Design}

Retrospective observational cohort study to measure the association between multiple exposures (BAMIC grade, muscle affected) and outcomes (TRFT, recurrence rate). The study was designed and reported according to the Strengthening the Reporting of Observational studies in Epidemiology statement. ${ }^{13}$

\section{Setting}

Data were collected from one English Premier League club through an electronic medical record system.

\section{Box 1 MRI thigh protocol parameters}

MRI thigh protocol

- t1 stir sag: FOV $420 \mathrm{~mm}$, matrix resolution $320 \times 320,5 \mathrm{~mm}$ slice thickness.

t1 coronal: FOV 400, matrix resolution $384 \times 384,5 \mathrm{~mm}$ slice thickness.

- t1 stir axial: FOV 220, matrix resolution $320 \times 320,6 \mathrm{~mm}$ slice thickness.

- pd axial: FOV 220, matrix resolution $384 \times 384,6$ mm slice thickness.

pd axial fs: FOV 220, matrix $320 \times 320,6 \mathrm{~mm}$ slice thickness.

\section{Participants}

All first team male players under contract across eight competitive seasons (English Premier League 20112016 and The English Football League Championship 2016-19) were eligible; including all injuries reported between August 2011 and June 2019, with acute pain to the posterior thigh, tenderness on hamstring palpation, contraction or function and an MRI examination performed within 7 days of injury. Direct muscle contusions were excluded based either on clinical presentation or imaging appearances.

\section{Data sources}

A retrospective review was performed of: injury date, age of player at time of injury, clinical diagnosis, TRFT, MRI scan date and injury recurrence to the same hamstring muscle during rehabilitation (exacerbation before TRFT) or within 3 months of TRFT. Consistent with the Pollock et $a t^{6}$ retrospective study, a recurrence was recorded if the player sustained an acute, sudden exacerbation of hamstring pain during exercise, followed by worsening of clinical tests and requiring cessation of current activity of rehabilitation or training for greater than 48 hours. Recurrence was categorised as yes/no. All MRI examinations were performed using a 1.5T MRI scanner (Siemens MAGNETOM Aera) using the same MRI thigh protocol at the same imaging centre (box 1).

\section{Bias}

A retrospective review of each MRI was independently performed by two fellowship trained musculoskeletal radiologists according to BAMIC using the same protocol (Pollock $e t a l$ ). If the HMI affected $\geq 1$ muscle, the muscle with the most extensive pathology determined by the injury details was considered the primary injured muscle. ${ }^{14}$ Any discrepancies were discussed and consensus reached regarding classification. MRI data collected included: scan date, primary muscle affected, second muscle affected, BAMIC (anatomical location and injury extent) and location (figure 1). All rehabilitation was provided by the Club's medical team and guided by its philosophy of progressive, functional and strength-based rehabilitation alongside graduated functional drills and running, limited by pain. The transition process to full team training was gradual and integrated 


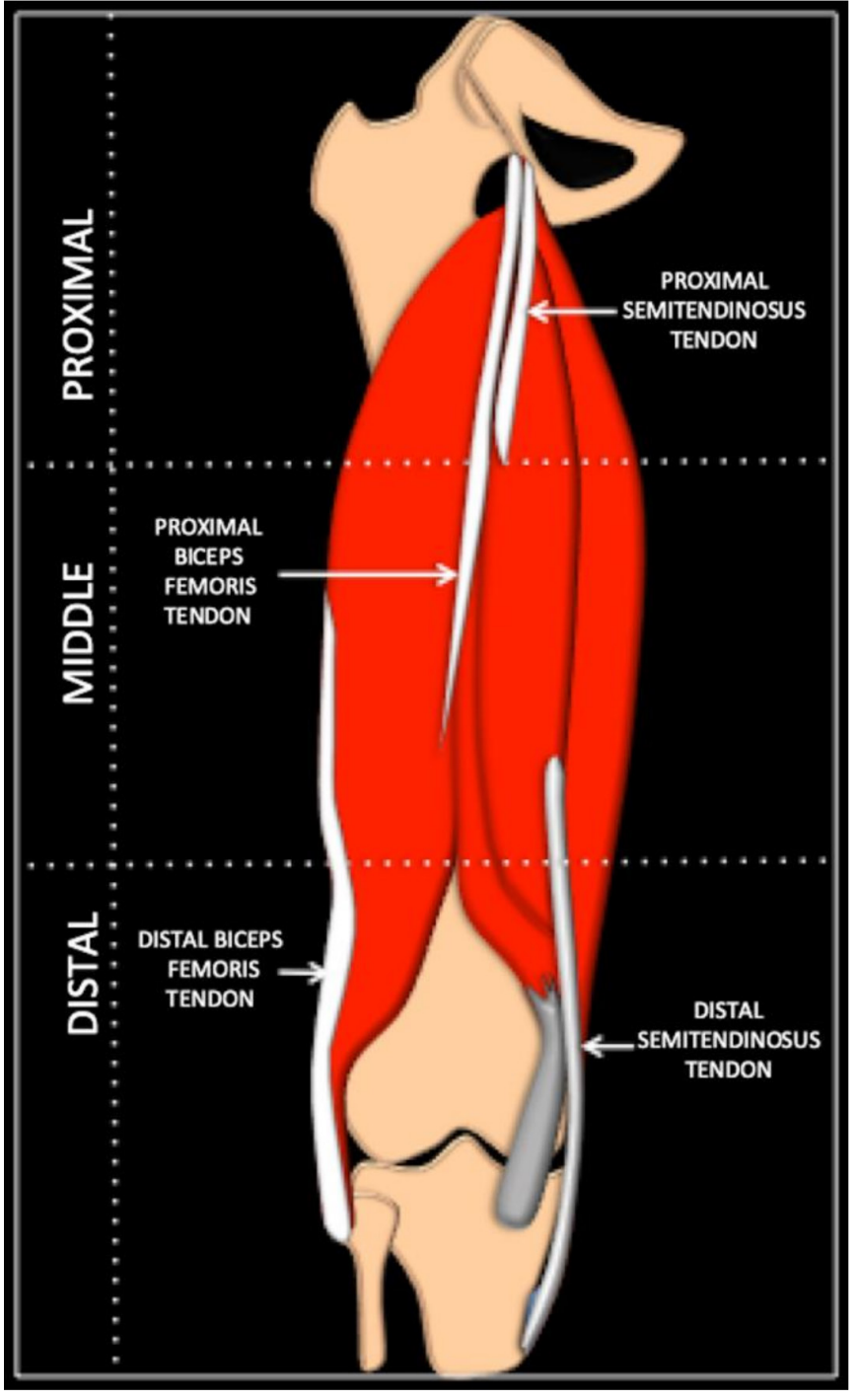

Figure 1 A schematic diagram of the left hamstring muscle divided into proximal, middle and distal third areas. It demonstrates the combined, approximate, free and intramuscular tendon lengths of the proximal and distal biceps femoris and semitendinosus muscle. The semimembranosus tendons are not illustrated.

to coach-led sessions with TRFT recorded following consensus between clinicians.

\section{Statistical methods}

Data were analysed consistent with Pollock et $a t^{6}$ as far as possible to enable comparison. Applying a quasi-experimental design, the relationship between independent (BAMIC) and dependent variables (TRFT, recurrence) was evaluated using the Kruskal-Wallis (for continuous TRFT outcome data) and Fisher's exact test (for two nominal variables for example, second muscle injured yes/no). For analysis of anatomic site $(a-c) 0$ injuries were excluded as they represent normal MRIs for $a$, and either normal or characteristic MRIs for delayed onset muscle soreness for b. All tests were nonparametric taking into account the nature of the BAMIC (not ordinal/ratio level data). Fischer's exact test was able to accommodate for the small anticipated sample size characteristic of elite sport populations (small numbers anticipated in cells of contingency tables). The null hypotheses were as follows:

H0-There is no association between TRFT and BAMIC.

$\mathrm{H} 0$ - There is no association between recurrence and BAMIC.

$\mathrm{H} 0$ - There is no association between TRFT, recurrence and injured muscle characteristics.

Additional descriptive analysis, not incorporated in BAMIC, explored anatomical injury location (proximal, middle, distal third from the sagittal and coronal images) of specific muscles and tendon (proximal, distal) involved (figure 1). In contrast to Pollock et $a l,{ }^{6}$ linear and logistic regression analyses were not used as assumptions for testing were not met (linearity of variables, multivariate normality, multicollinarity, inadequate sample size). The level of significance was set at $\mathrm{p}<0.05$. All analyses were performed using IBM SPSS Statistics, V.26.

\section{Ethical considerations}

Written authorisation and permission to access data was agreed by the Football Club. Participant data were stored under an allocated personal number with no identifying features (eg, date of birth). To ensure that no participant can be identified, data are presented in an unattributable format or at an aggregate level. Data are confidentially and securely stored for 10 years in line with research governance procedures.

\section{Patient involvement}

No players were involved in the conduct of the study.

\section{RESULTS}

\section{Participants}

Thirty-six elite football players were included with a median (IQR; range) age of 25 (6; range 18-36) years. Twelve participants experienced $\geq 2$ separate HMIs.

\section{Descriptive data}

There were 61 HMIs from the 36 players. Median (IQR; range) TRFT following HMI was 18 (14; 2-103) days. The most common muscle injured was biceps femoris $(n=40)$ (table 1).

\section{BAMIC of injuries}

Table 2 details the TRFT as median (IQR, range) for each BAMIC. Reinjury is presented as a percentage for each classification. Out of $61 \mathrm{HMI}, \mathrm{n}=13$ (21.3\%) are BAMIC 'c' (intramuscular tendon) injuries.

\section{BAMIC and TRFT}

There was a statistically significant difference in TRFT for BAMIC $\left(\chi^{2}=20.03, \mathrm{p}=0.006, \mathrm{df}=7\right)$ with a mean rank TRFT of 16 days for BAMIC 0a, 23 days BAMIC 0b, 12 days BAMIC 1a, 29 days BAMIC 1 b, 23 days BAMIC 2a, 37 days BAMIC 2b, 46 days BAMIC 2c and 33 days BAMIC $3 \mathrm{c}$ There were only three injuries with BAMIC grade 3c. 
Table 1 Descriptive data of the study participants and the hamstring injuries

\begin{tabular}{ll}
\hline Variable & $\begin{array}{l}\text { Descriptive } \\
\text { value }\end{array}$ \\
\hline Study participants & 61 \\
\hline Median age in years (IQR, range) & $25(6.00 ; 18-36)$ \\
\hline Players who sustained $\geq 2$ HMI (n) & $12(2-7$ injuries) \\
\hline Total no of injuries by specific muscle* (\%) \\
\hline Biceps femoris n (\%) & $40(65.6)$ \\
\hline Semitendinosus n (\%) & $7(11.5)$ \\
\hline Semimembranosus n (\%) & $10(16.4)$ \\
Median TRFT in days (IQR, range) & $18(14.00 ;$ \\
Reinjury (\%) & $2-103)$ \\
Yes, reinjury & $10(16.4)$ \\
Yes, exacerbation & $6(9.8)$ \\
\hline
\end{tabular}

${ }^{*}$ Note $\mathrm{n}=4$ grade 0 a injuries

$\mathrm{HMI}$, hamstring muscle injury; TRFT, time to return to full training.

Figure 2 illustrates the relationship between BAMIC and TRFT.

BAMIC was explored further regarding grading and anatomical sites within muscle (a,b or c) components separately. There was a statistically significant difference in TRFT for BAMIC severity $(0-3)\left(\chi^{2}=11.52, \mathrm{p}=0.009\right.$, $\mathrm{df}=3$ ), with a mean rank TRFT of 19 days for BAMIC 0 , 25 days BAMIC 1, 39 days BAMIC 2 and 33 days BAMIC 3.

Table 2 TRFT and reinjury rate of HMI according to British Athletic Muscle Injury Classification

\begin{tabular}{lcll}
\hline $\begin{array}{l}\text { British } \\
\text { Athletic } \\
\text { Muscle Injury } \\
\text { Classification }\end{array}$ & $\begin{array}{l}\text { No of } \\
\text { injuries }\end{array}$ & $\begin{array}{l}\text { Median TRFT (IQR; } \\
\text { range) days }\end{array}$ & $\begin{array}{l}\text { Reinjury } \\
\mathbf{n}(\%)\end{array}$ \\
\hline Oa & 4 & $8.00(13.75 ; 5-22)$ & N/A \\
\hline Ob & 4 & $13.00(13.75 ; 7-22)$ & $1(25)$ \\
\hline 1a & 6 & $8.00(9.00 ; 2-14)$ & 0 \\
\hline 1b & 15 & $17.00(12.00 ; 3-34)$ & $1(7)$ \\
\hline 2a & 3 & $12.00\left({ }^{*} ; 3-25\right)$ & 0 \\
\hline 2b & 16 & $22.00(14.5 ; 6-39)$ & $3(19)$ \\
\hline 2c & 10 & $37.00(43.00 ; 8-103)$ & $4(40)$ \\
\hline 3a & & & \\
\hline 3b & & & $1(33)$ \\
\hline 3c & 3 & $15.00\left({ }^{*} ; 9-63\right)$ & \\
\hline 4 & & & \\
\hline 4c & 61 & $18.00(14.00 ; 2-103)$ & $10(16)$ \\
\hline Total & & & \\
\hline
\end{tabular}

${ }^{*}$ Not possible to calculate IQR $(\mathrm{n}=3)$

$\mathrm{HMI}$, hamstring muscle injury; N/A, not available; TRFT, time to return to full training.

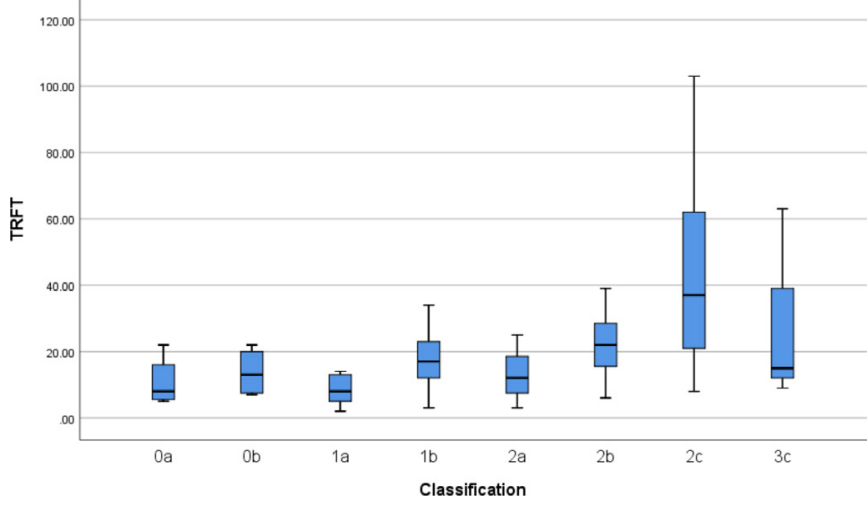

Figure 2 British Athletic Muscle Injury Classification of hamstring injuries and median (IQR; range) time (days) to return to full training (TRFT).

There was also a statistically significant difference in TRFT for anatomical site BAMIC $(\mathrm{a}-\mathrm{c})$ excluding grade $0\left(\chi^{2}=12.68, \mathrm{p}=0.002, \mathrm{df}=2\right)$, with a mean rank TRFT of 12 days for BAMIC 'a', 27 days BAMIC 'b' and 36 days BAMIC 'c'. In particular, it can be concluded that TRFT for HMIs with intramuscular tendon involvement (anatomical site, 'c') was statistically significantly higher than HMIs with no intramuscular tendon involvement (sites ' $a$ ' and ' $b$ ') $\left(\chi^{2}=6.16, \mathrm{p}=0.013, \mathrm{df}=1\right)$ with a mean rank TRFT of 24 days for no tendon involvement and 36 days for tendon involvement.

\section{Injured muscle, second muscle involvment and TRFT}

There was a statistically significant difference in TRFT for injured muscle $\left(\chi^{2}=6,23, \mathrm{p}=0.044, \mathrm{df}=2\right)$, with a mean rank TRFT of 18 days for semimembranosus, 27 days for semitendinosus, and 32 days for biceps femoris (table 3 ). Fisher's exact test enabled assessment of the differences of BAMIC according to injured muscle. There was a significant difference $\left(\chi^{2}=39.60, \mathrm{p}=0.000\right)$ between BAMIC and injured muscle.

Only data for Biceps femoris HMIs were possible to analyse descriptively. Of the $n=11$ BAMIC $1 \mathrm{~b}$ biceps femoris (long head) HMIs, three were proximal, three were middle and five were distal third injuries. Of the $\mathrm{n}=102 \mathrm{~b}$ biceps femoris (long head) HMIs, four were proximal, two were middle and four were distal third injuries. Of the $n=10$ 2c long head HMIs, two were proximal, two were middle and six were distal third injuries; with five distal third injuries involving the distal tendon and four involving a second muscle-short head biceps femoris.

Twenty HMIs involved a second muscle (32.8\%), of which $75 \%(n=15)$ were injuries to biceps femoris. All grade 1 or above HMIs involving a second muscle were BAMIC 'b' (musculotendinous junction) or 'c' (intramuscular tendon).

TRFT for HMIs with second muscle involvement was statistically significantly higher than HMIs with no second muscle involvement $\left(\chi^{2}=19.161, \mathrm{p}=0.000, \mathrm{df}=1\right)$ with a mean rank TRFT of 42 days for second muscle involvement and 22 days for none. 
吝
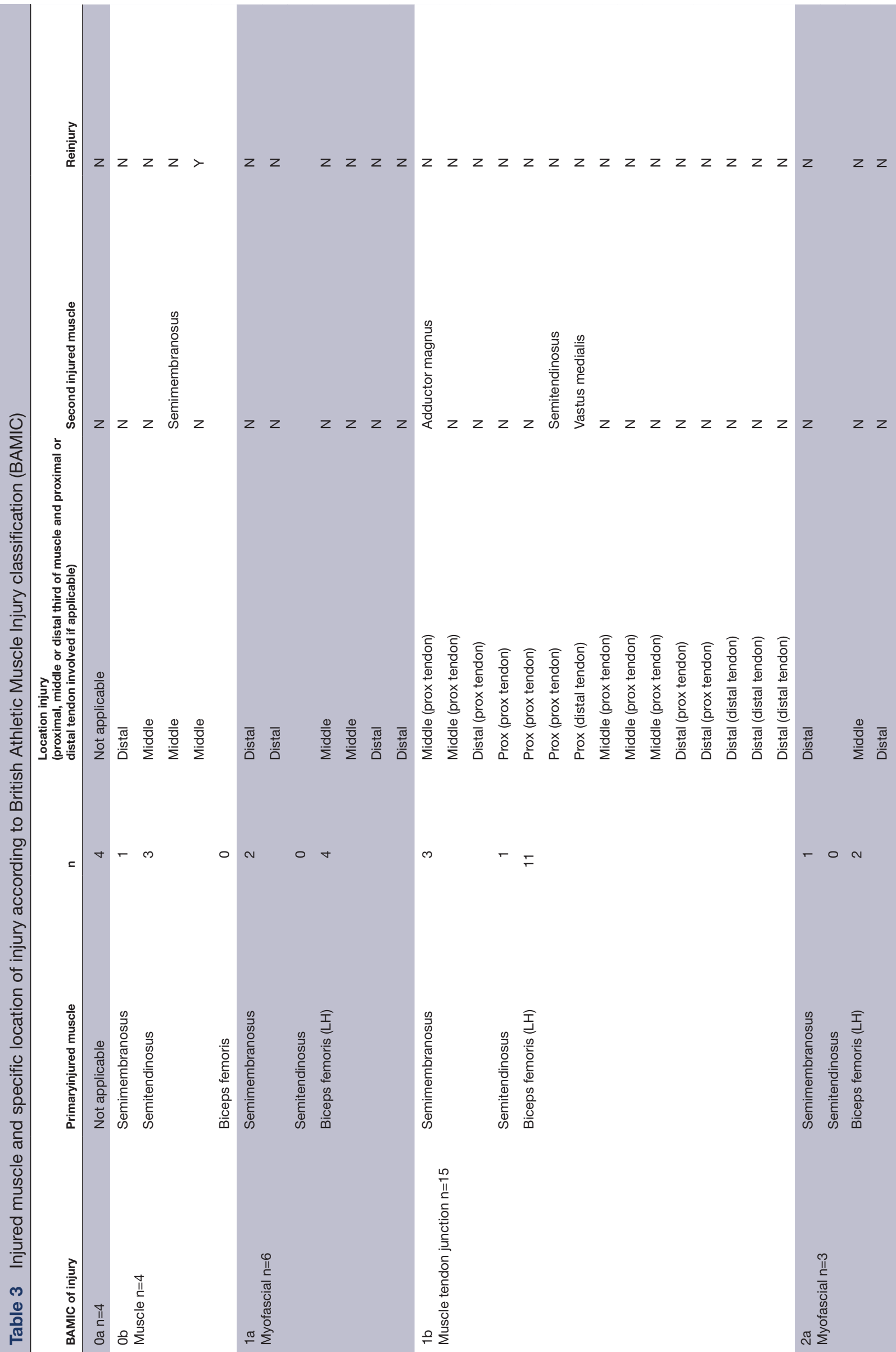


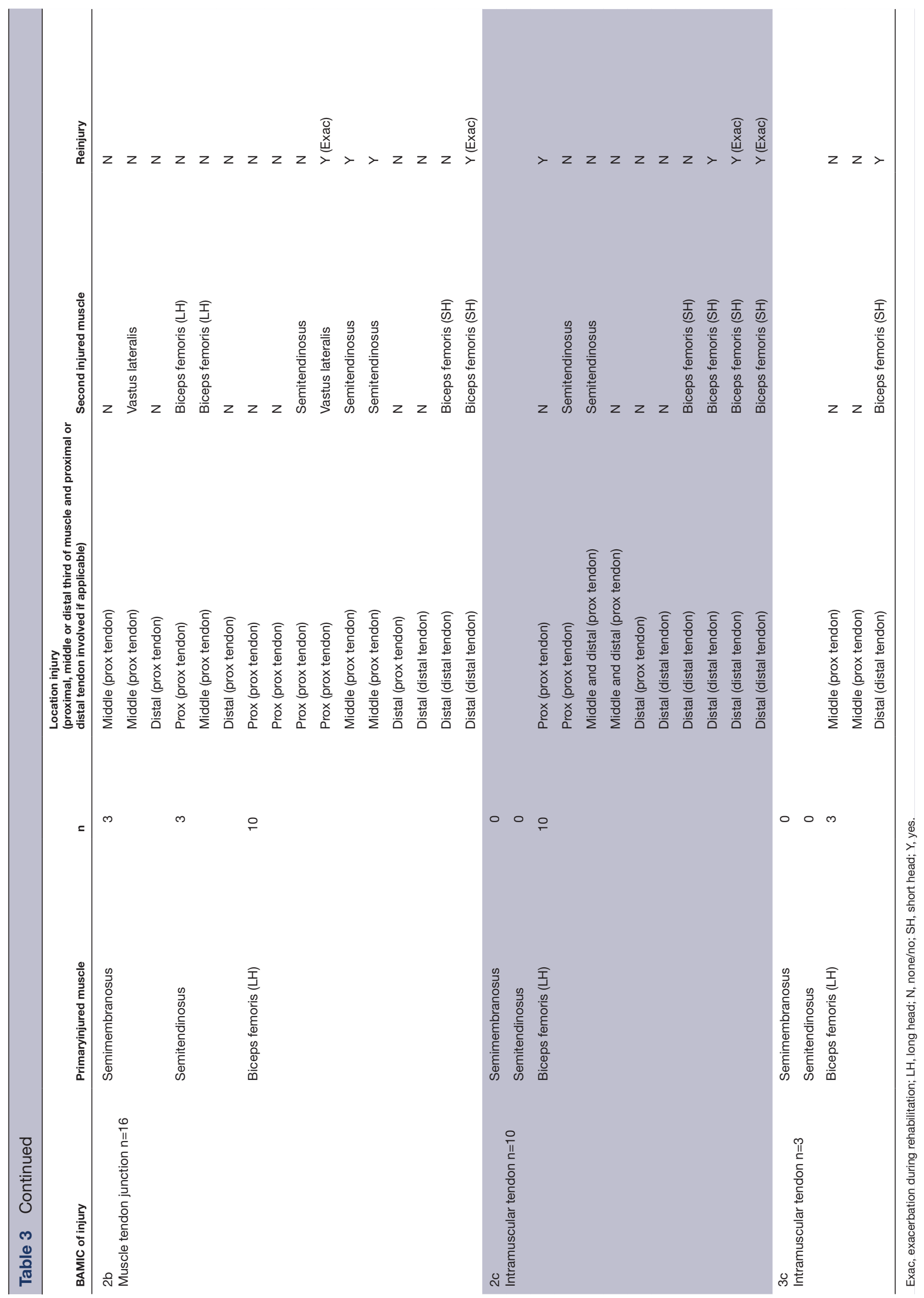




\begin{tabular}{|c|c|c|}
\hline $\begin{array}{l}\text { BAMIC according to site } \\
\text { (a-c), excluding grade } 0\end{array}$ & No of injuries & Reinjury n (\%) \\
\hline a & 9 & 0 \\
\hline b & 31 & 5 (16.1) \\
\hline c & 13 & 5 (38.5) \\
\hline
\end{tabular}

BAMIC, British Athletic Muscle Injury Classification; HMI, hamstring muscle injury.

\section{BAMIC and reinjury}

Ten cases $(16.4 \%)$ of HMI reinjury, included four cases $(40.0 \%)$ of HMI exacerbation before TRFT. Reinjury rate involving the intramuscular tendon ('c') was $38.5 \%$ (table 4). In comparison, reinjury without extension into the intramuscular tendon ('a', 'b'), excluding grade 0 injuries, was $12.5 \%$.

Overall, there was no significant difference $\left(\chi^{2}=10.38\right.$, $\mathrm{p}=0.083$ ) within BAMIC according to reinjury. When looking at BAMIC regarding grade severity $(0-4)$ and site $(\mathrm{a}, \mathrm{b}, \mathrm{c})$ components separately, there was a significant difference $\left(\chi^{2}=8.52, \mathrm{p}=0.024\right)$ within BAMIC severity reinjury and $\left(\chi^{2}=6.47, \mathrm{p}=0.028\right)$ within BAMIC site classification according to reinjury. In particular, there was a significant difference $\left(\chi^{2}=5.64, \mathrm{p}=0.031\right)$ between intramuscular tendon (site 'c') vs no intramuscular tendon involvement (site 'a' and 'b') and reinjury.

\section{Injured muscle, second muscle and reinjury}

There was no significant difference $\left(\chi^{2}=2.94, \mathrm{p}=0.368\right)$ between reinjury and injured muscle. There was a significant difference $\left(\chi^{2}=10.74, \mathrm{p}=0.002\right)$ between a second muscle being involved (yes or no) and reinjury.

There were sufficient data to descriptively analyse location of injury for biceps femoris HMIs, with most injuries with reinjury involving the distal third section and distal tendon site ( $\mathrm{n}=5 ; 55.6 \%$; $\mathrm{n}=1$ 2b HMI, $\mathrm{n}=3$ 2 c HMI, $\mathrm{n}=1$ 3c HMI). All five reinjuries involving this distal third section and distal tendon had evidence of injury to both the long and short heads of biceps femoris. Notably, three occurred before TRFT (ie, exacerbation during rehabilitation) and represented three out of the four exacerbations.

\section{DISCUSSION}

HMIs with any degree of intramuscular tendon involvement are associated with increased TRFT (mean rank of 36 days with tendon involvement vs 24 days without) and an increased risk of reinjury (38.5\% reinjury with tendon involvement vs $12.5 \%$ without) in elite footballers. This study also highlights potential significance of injury to the DMTJ of the biceps femoris.

\section{TRFT in HMI with intramuscular tendon involvement}

Similar to Pollock et al, ${ }^{6}$ this study shows that HMIs in elite footballers extending into the tendon (' $c$ ') are associated with an increased TRFT and reinjury rate. The degree of

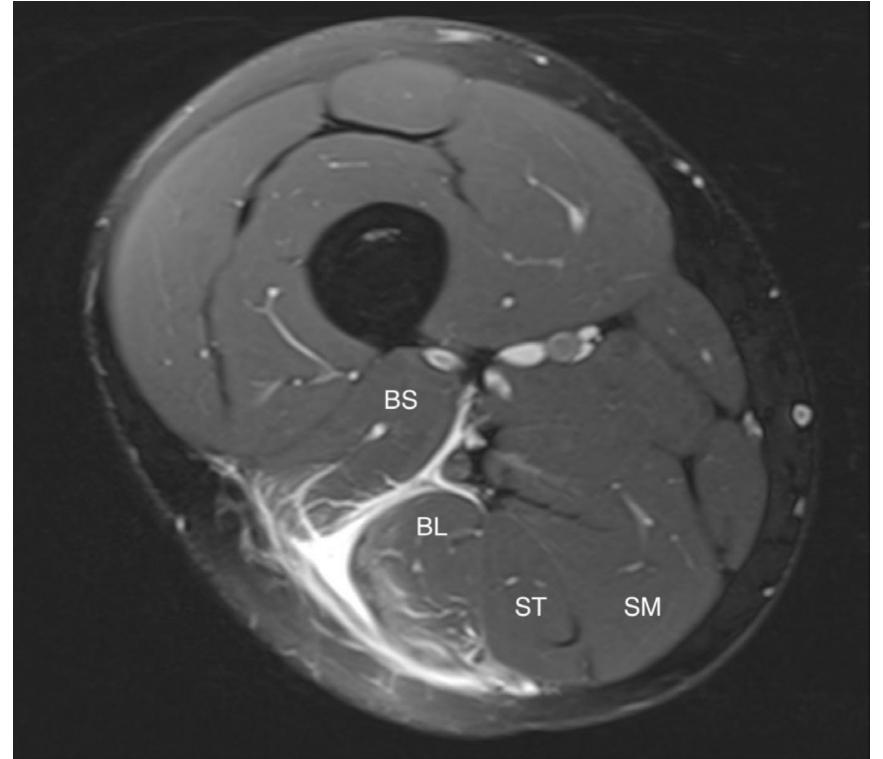

Figure 3 Axial proton density fat suppressed MRI image of an injury to the distal musculotendinous junction involving both the short head (BS) and long head (BL) of the biceps femoris. The semitendinosus (ST) and the semimembranosus (SM) is normal.

intramuscular tendon involvement on the TRFT seems less clear across other studies. While it is not ideal to compare medians with means, the trends are important to discuss. Players with $2 \mathrm{c}$ and $3 \mathrm{c}$ injuries in this study had a median TRFT of 37 days and 15 days, respectively. In contrast, Pollock et at reported a mean TRFT of 27 days for 2c which increased to 84 days for $3 \mathrm{c}$ injuries. The prospective study of van der Made $e t a l^{11}$ did not use BAMIC per se, but a comparison is possible as they did report injuries according to the separate degrees of intramuscular tendon involvement. They report a mean TRFT of 24 days in the group equivalent to a BAMIC grade $2 \mathrm{c}$ and a mean TRFT of 25 days rising to 30 days, for additional signs of tendon waviness/loss of tension, which would be classed as BAMIC grade 3c. It can, therefore, be deduced that the median TRFT of 37 days for the BAMIC 2c injuries in this study are considerably higher than those seen in the other studies. With regards to BAMIC $3 \mathrm{c}$ injuries, our median TRFT of 15 days, is closer to the TRFT reported by van der Made $e t a l,{ }^{11}$ but considerably shorter to that reported by Pollock et al (84 days). ${ }^{6}$ It is acknowledged however that there are low numbers of BAMIC grade 3c injuries in this current study.

The differences are multifactorial and require further evaluation beyond the extent of intramuscular tendon involvement where there is a wide range of TRFT in both 2c (8-103 days) and 3c (9-63 days) injuries in our study. Anatomical injury location, which is not routinely categorised by MRI classification beyond site within the muscle, cannot differentiate all possible important clinical entities which may result in the differences reported between studies. Entwisle $e t a l^{12}$ have described the DMTJ of the biceps femoris as a distinct clinical entity and report a 


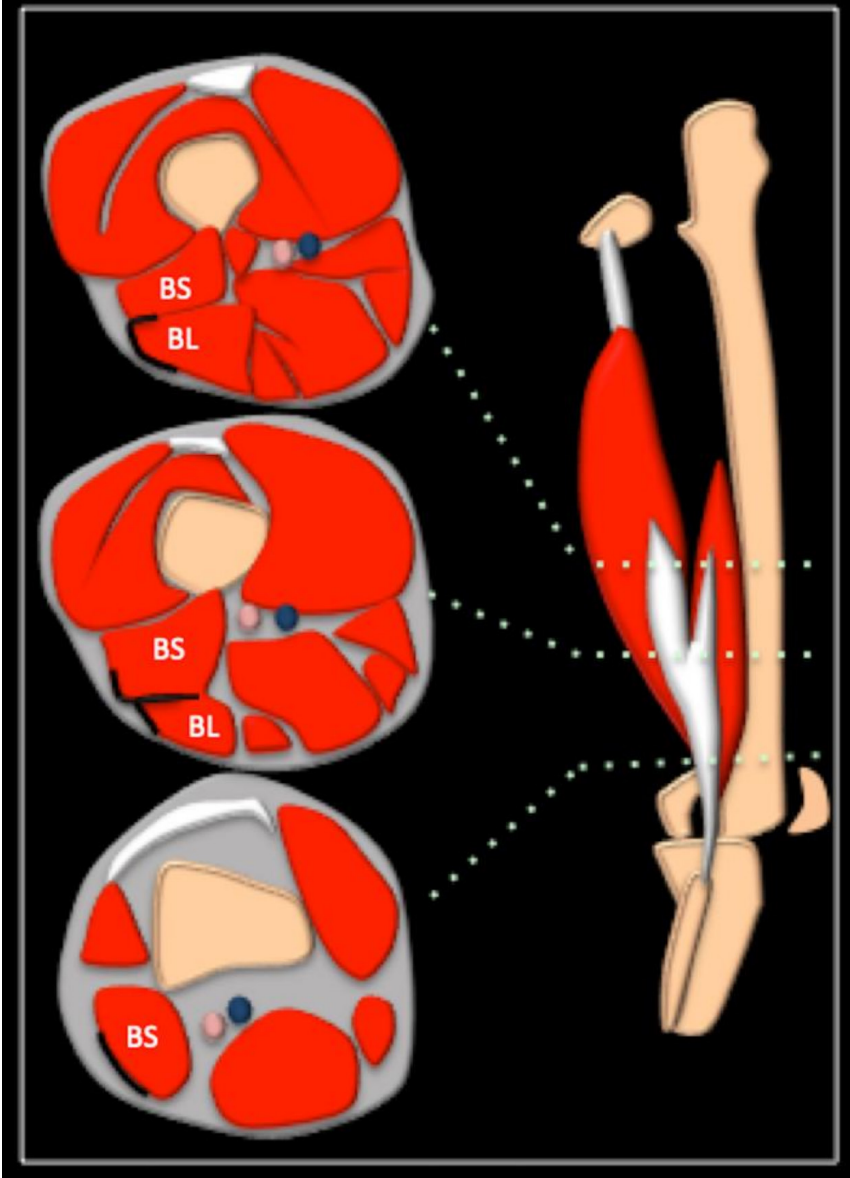

Figure 4 Schematic diagram to demonstrate the sequential axial anatomy of the distal musculotendinous junction of the long (BL) and short (BS) head of the biceps femoris. The middle axial image shows the DMTJ as a T-shaped structure. DMTJ, distal musculotendinous T junction.

reinjury rate of $54 \%$ in Australian football players. We show increased reinjury at the distal biceps femoris related to the distal tendon, described and termed DMTJ by Entwisle $e t a l,{ }^{12}$ involving both the long and short head (figure 3). This area of the biceps femoris has complex anatomy where two heads (long and short), with their different sites of origin and separate innervation, give rise to opposing force vectors during contraction that converge on and transmit through the DMTJ (figure 4). ${ }^{12}$ In this study, three out of four exacerbations during graded rehabilitation occurred at this location and may suggest that a different approach, possibly beyond clinical progression criteria are needed. Interval MRI may be of value to monitor scar formation and maturation of this aponeurotic, as opposed to a cord-like, intramuscular tendon appearance seen at this location. ${ }^{12}$ Of note, intramuscular tendon appearance (aponeurotic, cordlike) varies according to hamstring location, and between individuals, and with intramuscular tendon not behaving like a free tendon functionally or when injured, the effect of the varying intramuscular tendon appearances are not known. ${ }^{15}$ These factors warrant further study that may help us understand the wide range of TRFT seen with varying degrees of intramuscular tendon injury reported in studies.

\section{Contextual factors}

The type of sport (eg, football, sprinting, squash, rugby) and level of participation for the athlete is also a key consideration when comparing differences between studies. Return to train is a continuum, paralleled with recovery and rehabilitation. ${ }^{16}$ Some individuals will return to full squad training earlier than other players with similar injury classification. TRFT in team sports, such as football, will vary according to position in team, position on the field as well as other common contextual circumstances such as upcoming competition, timing in season and social and financial costs. Our study reports TRFT in high elite level first team English football and should therefore be considered more applicable to the HMIs seen in this population.

\section{Limitations}

Our cohort includes professional level first team football players only. While potentially increasing the external validity and clinical relevance for similar football players at this level, the lack of a sufficient number of injuries within each category limits our statistical analyses. In particular, there are a low number of BAMIC grade 3c vs 2c injuries. We are unable to comment on the importance of intramuscular tendon injuries of the semimembranosus and semitendinosus as no ' $c$ ' injuries to these muscles were seen in our study. The clinicians involved in the decision for TRFT were not blinded to the MRI findings although reference to the specific BAMIC was not used by clinicians in the first four seasons of data collection. All images were acquired by a $1.5 \mathrm{~T}$ MRI and greater diagnostic accuracy might be achieved by 3.0T MRI.

\section{CONCLUSION}

This study provides evidence that HMIs in elite footballers, involving the intramuscular tendon increases TRFT and risk of reinjury. The use of BAMIC can, therefore, provide useful information for the managing clinician. However, this study shows that while knowledge of the intramuscular tendon involved in injury is helpful, considering other anatomical entities may help refine the wide range of TRFT seen with intramuscular tendon involvement. We describe the potential importance of recognising injury to the DMTJ of the biceps femoris involving both the long and short heads.

\section{Twitter Ricky Shamji @p4exercise}

Acknowledgements John Hartley, Rob Marshall and Steve Polley provided invaluable insight during the process.

Contributors RS, AR and KAK were involved in the conception and design of this study; RS and KAK were involved in the acquisition of data; RS, AR and SLJJ conducted the analyses; all authors were involved in interpretation of the data; $\mathrm{RS}$ and $\mathrm{AR}$ prepared the first draft and all authors critically reviewed and revised this several times, with addition of important intellectual content; RS, RB and SLJJ produced the illustrations; all authors gave final approval of the version to be submitted. 
Funding The authors have not declared a specific grant for this research from any funding agency in the public, commercial or not-for-profit sectors.

Competing interests None declared.

Patient and public involvement Patients and/or the public were not involved in the design, or conduct, or reporting, or dissemination plans of this research.

Patient consent for publication Not required.

Ethics approval Ethics committee of University of Birmingham.

Provenance and peer review Not commissioned; externally peer reviewed.

Data availability statement № data are available. All data are reported.

Open access This is an open access article distributed in accordance with the Creative Commons Attribution Non Commercial (CC BY-NC 4.0) license, which permits others to distribute, remix, adapt, build upon this work non-commercially, and license their derivative works on different terms, provided the original work is properly cited, appropriate credit is given, any changes made indicated, and the use is non-commercial. See: http://creativecommons.org/licenses/by-nc/4.0/.

ORCID iD

Ricky Shamji http://orcid.org/0000-0001-8224-8357

\section{REFERENCES}

1 Ekstrand J, Hägglund M, Waldén M. Epidemiology of muscle injuries in professional football (soccer). Am J Sports Med 2011;39:1226-32.

2 de Visser HM, Reijman M, Heijboer MP, et al. Risk factors of recurrent hamstring injuries: a systematic review. Br J Sports Med 2012;46:124-30.

3 Ekstrand J, Lee JC, Healy JC. MRI findings and return to play in football: a prospective analysis of 255 hamstring injuries in the UEFA elite Club injury study. Br J Sports Med 2016;50:738-43.

4 Comin J, Malliaras P, Baquie P, et al. Return to competitive play after hamstring injuries involving disruption of the central tendon. $A m \mathrm{~J}$ Sports Med 2013;41:111-5.

5 Brukner P, Connell D. 'Serious thigh muscle strains': beware the intramuscular tendon which plays an important role in difficult hamstring and quadriceps muscle strains. Br J Sports Med 2016:50:205-8.

6 Pollock N, Patel A, Chakraverty J, et al. Time to return to full training is delayed and recurrence rate is higher in intratendinous ('c') acute hamstring injury in elite track and field athletes: clinical application of the British Athletics Muscle Injury Classification. Br J Sports Med 2016:50:305-10.

7 Slavotinek JP, Verrall GM, Fon GT. Hamstring injury in athletes: using MR imaging measurements to compare extent of muscle injury with amount of time lost from competition. AJR Am J Roentgenol 2002;179:1621-8.

8 Connell DA, Schneider-Kolsky ME, Hoving JL, et al. Longitudinal study comparing sonographic and MRI assessments of acute and healing hamstring injuries. AJR Am J Roentgenol 2004;183:975-84

9 Pollock N, James SLJ, Lee JC, et al. British athletics muscle injury classification: a new grading system. $\mathrm{Br} J$ Sports Med 2014:48:1347-51.

10 Crema MD, Guermazi A, Reurink G, et al. Can a clinical examination demonstrate intramuscular tendon involvement in acute hamstring injuries? Orthop J Sports Med 2017;5:232596711773343.

11 van der Made AD, Almusa E, Whiteley R, et al. Intramuscular tendon involvement on MRI has limited value for predicting time to return to play following acute hamstring injury. Br J Sports Med 2018;52:83-8

12 Entwisle T, Ling Y, Splatt A, et al. Distal Musculotendinous T junction injuries of the biceps femoris: an MRI case review. Orthop J Sports Med 2017;5:232596711771499.

13 von Elm E, Altman DG, Egger M, et al. The strengthening the reporting of observational studies in epidemiology (STROBE) statement: guidelines for reporting observational studies. Lancet 2007;370:1453-7.

14 Hallén A, Ekstrand J. Return to play following muscle injuries in professional footballers. J Sports Sci 2014;32:1229-36.

15 Brukner P, Cook JL, Purdam CR. Does the intramuscular tendon act like a free tendon? Br J Sports Med 2018;52:1227-8.

16 Ardern CL, Glasgow P, Schneiders A, et al. 2016 consensus statement on return to sport from the first world Congress in sports physical therapy, Bern. Br J Sports Med 2016;50:853-64. 